\title{
Sobre la importancia de la internacionalización en universidades de clase mundial, de acuerdo con ránquines de universidades
}

\author{
On the Importance of Internationalization in World-Class Universities, \\ According to University Rankings
}

\author{
Darío Liberona ${ }^{1}$
}

Para citar este artículo: Liberona, D. (2019). Sobre la importancia de la internacionalización en universidades de clase mundial, de acuerdo con ránquines de universidades. Revista ObIES, 3, 26-37.

Recibido: 3-septiembre-2019 / Aceptado: 20-noviembre-2019

\section{Resumen}

En los últimos años la internacionalización de las universidades se ha hecho cada vez más importante debido a la globalización de nuestra economía y sociedad. Para las universidades de clase mundial la colaboración y, por ende, la internacionalización resultan fundamentales. Las universidades forman el capital humano de la sociedad y las naciones. En la actualidad la revolución industrial 4.0, las redes sociales, la inteligencia artificial, la internet de las cosas y la transformación digital son todas tendencias que se relacionan con la globalización y requieren que las universidades aumenten sus niveles de internacionalización. Existen muchas universidades que solicitan experiencias internacionales obligatorias de todos sus alumnos (pasantías de estudios o prácticas laborales en otros países); en el caso de Latinoamérica estamos rezagados en este proceso de internacionalización. Este trabajo muestra algunos factores importantes de la internacionalización y el grado de importancia que le asignan los ránquines internacionales de universidades. Las universidades latinoamericanas que quieran mejorar su impacto deben mejorar sus procesos y resultados de internacionalización, como los casos actuales de las universidades chilenas y brasileñas, las cuales lideran este proceso en Latinoamérica. Para Ilegar a esto, se necesita de mayores incentivos y el apoyo de los gobiernos en los procesos de internacionalización y de colaboración en la investigación.

Palabras clave: universidades de clase mundial; ránquines de universidades; internacionalización; benchmarking de universidades.

\begin{abstract}
In recent years the internationalization of universities has become increasingly important due to the globalization of our economy and society. For worldclass universities, collaboration and, therefore, internationalization are fundamental. Universities provide the human capital of society and nations. Today the industrial revolution 4.0, social networks, artificial intelligence, the internet of things and digital transformation are all trends that are related to globalization and require universities to increase their levels of internationalization. There are many universities that request obligatory international experiences from all their students (internships or
\end{abstract}

1 PhD. Dirección General de Empresas de la Universidad de Lleida (España). MBA de la Universidad de Lleida (España) y de la Universidad de Santiago (Chile). Ingeniero Electrónico mención telecomunicaciones de la Universidad Técnica Federico Santa María (USM, Chile). Ejecutivo Profesional de amplia trayectoria gerencial, durante los últimos 16 años ha desempeñado cargos gerenciales en los Bancos de Chile, Bice y Edwards. Especializado en materias de marketing, desarrollo de negocios, ventas, e-commerce y administración de empresas. Hoy es Director Procesos en Benchmarking de la Universidad Técnica Federico Santa María (USM, Chile). Correo electrónico: dario.liberona@usm.cl 
internships in other countries); in the case of Latin America we are lagging behind in this process of internationalization. This work shows some important factors of internationalization and the degree of importance assigned to it by the international rankings of universities. Latin American universities that want to improve their impact must improve their internationalization processes and results, such as the current cases of Chilean and Brazilian universities, which lead this process in Latin America. To achieve this, greater incentives and support from governments in the processes of internationalization and collaboration in research are needed.

Keywords: world-class universities; university rankings; internationalization; university benchmarking.

\section{¿Qué hace a una universidad una universidad de clase mundial?}

Simmons (2003) ofrece una amplia visión de los principios que deben cumplirse si se considera que una universidad es una institución de clase mundial. El primer principio sugiere que la misión y la visión de las universidades deben estar alineadas con los objetivos sociales locales. El segundo se centra en la calidad y el rigor académico, especialmente en lo que respecta a la revisión por pares académicos y la generación de nuevo conocimiento. El principio final sugiere que la institución debe ser progresista, afrontar el futuro y alimentar la democracia, sobre todo a través de la libertad académica y el fomento de ideas libres.

Salmi sugiere que los resultados superiores de las universidades de clase mundial se pueden atribuir a "tres factores complementarios en juego en las mejores universidades" (2009, p. 19), a saber, una alta concentración de talento de académicos y alumnos, abundantes recursos y un gobierno favorable.

En general existe consenso en que las universidades de clase mundial cuentan con cinco aspectos generales:
1. Alumnos de calidad.

2. Profesores de calidad.

3. Investigación con alto impacto, internacional y multidisciplinaria.

4. Internacionalización, gran cantidad de alumnos y profesores extranjeros. Trabajo colaborativo.

5. Recursos financieros y físicos. Colaboración con las empresas.

Otra característica es que se trata de universidades reconocidas y con gran reputación.

Toda nación que desee avanzar en la senda del desarrollo y progreso requiere de más universidades de clase mundial. Es por esta razón por la que muchos gobiernos han desarrollado estrategias y planes para poder desarrollarlas, entre ellos: Japón, Rusia, Turkia, Taiwán, China; estas universidades tienen como característica común el que colaboran en el desarrollo de la competitividad de sus respectivas naciones en una economía globalizada (Altbach, 2011).

\section{La internacionalización de las universidades}

Sobre la importancia de la internacionalización de las universidades, el grupo de investigación español Estudiasi, en su blog de studio (Studiasi, 2017), plantea que la internacionalización es, sobre todo, difusión y comunicación del conocimiento que se crea en el seno de la institución universitaria, dando importancia a la labor de colaboración internacional en la investigación; y, por otro lado, el intercambio de profesores y alumnos que dan cuenta del encuentro entre culturas.

Por su parte, el administrador de educación superior Hans de Wit nos plantea que la internacionalización de la educación superior ha pasado de una participación marginal a ser un componente fundamental de la estrategia de las universidades; de igual modo, que la internacionalización es más que la movilidad de alumnos de pregrado y que 
se requiere de una estrategia que tenga una visión integrada y amplia al interior de la universidad, con aspectos como desarrollo de marca (branding), ciudadanos globales, moocs, programas de doble título, intercambio cultural, y escalafones globales (Night y Wit, 2018).

Con respecto a los escalafones internacionales sobre universidades que han adquirido gran popularidad en los últimos años, en general valoran y ponderan como aspectos de la internacionalización tres factores fundamentales: la opinión de los pares académicos internacionales (encuestas), la colaboración entre investigadores, así como el intercambio de profesores y alumnos.

El número de alumnos internacionales ha aumentado de manera importante en los últimos años, desde 800000 alumnos de intercambio en 1975 a cerca de 5000000 el año 2015, de acuerdo con la OCDE. De esta situación se valora cada vez más la experiencia internacional de los estudiantes, las mejoras en el manejo de idiomas, las habilidades interculturales y el desarrollo de redes internacionales (Reynolds, 2018).

Otro gran beneficio de la internacionalización tiene que ver con la colaboración y las alianzas internacionales en una serie de materias, intercambios de profesores y proyectos de investigación conjuntos (Huang, 2018).

La internacionalización suele tener una justificación económica en términos de beneficios económicos a largo plazo como producto del intercambio de recursos humanos calificados (crecimiento económico, mercado laboral, comercio exterior, etc.). Pero también considera beneficios financieros a corto plazo en forma de generación de ingresos de estudiantes internacionales. Estos suelen ser los motivos más importantes para que todos los tipos de universidades se globalicen (Abbas y Yousafzai, 2018).

La internacionalización de la educación superior es actualmente parte importante del proceso de globalización, y ya no puede ser vista en el contexto del entorno nacional, hoy en día puede ser relacionada con la función completa de la universidad en vez de un aspecto especifico, y se hace necesaria una serie de estratégicas para poder abordar este proceso (Zha, 2003).

En este sentido, John Hudzik, destacado académico de Nafsa (Association of International Educators), plantea que pronto habrá más de siete millones de estudiantes internacionales (2025). Y que siendo la vocación de las universidades la creación y la difusión de ideas a través de la educación y su aplicación la internacionalización es fundamental para la misión de las universidades; asimismo define la internacionalización integral como "un compromiso, confirmado a través de la acción, para infundir perspectivas internacionales y comparativas a través de la enseñanza, la investigación y las misiones de servicio a la educación superior" (Hudzik, 2011)

\section{Sobre los ránquines internacionales de universidades}

No cabe duda de que los escalafones se han convertido en una parte importante del panorama de la educación superior, tanto a nivel mundial como local.

En este panorama, las clasificaciones han aumentado en importancia y han proliferado de manera inimaginable hace algunos años. Sin lugar a dudas, los ránquines desempeñan ahora un papel muy importante en la formación de las opiniones de los estudiantes actuales y potenciales, de los padres, de los empleadores y del gobierno acerca de la calidad de las instituciones de educación terciaria.

Hoy en día es común observar políticas y programas enteros de gobiernos aparentemente más preocupados por la posición en las clasificaciones que por la relevancia de sus instituciones de educación superior. A veces esto resulta en el desvío de una cantidad significativa de recursos a algunas instituciones, limitando al mismo tiempo el apoyo a otras. 
Existen numerosas críticas a los escalafones y su visión predefinida de una institución ideal, que no siempre toma en consideración las diferencias contextuales significativas. Algunos consideran que tiende a imponer una visión unilateral de una institución - en su mayoría una universidad tradicional orientada a la investigación y altamente selectiva- que no es necesariamente la más sensible a las diversas necesidades de las comunidades en donde se ubican estas instituciones. Sin embargo, los ránquines han seguido proliferando y ganando su poder de influencia.

La mayoría de las clasificaciones bien conocidas tienden a equiparar la calidad institucional con la productividad de la investigación, que se mide por el número e impacto de sus publicaciones en revistas con revisión por pares, o por la selectividad en sus procesos de admisión. Por supuesto, al considerar estos indicadores disminuye el número de las instituciones que ponen mayor énfasis en la enseñanza y de manera indirecta se promueve el principio de "publicar o perecer", importante en las universidades de mayor reputación. En la búsqueda de una mejor posición en los ránquines, lo más probable es que los fondos internos y externos tiendan a favorecer programas académicos o unidades de investigación que estén más inclinados a involucrarse en la dinámica de investigación y publicación. No obstante, en los últimos años ha aumentado la consideración del papel de otras funciones importantes de las instituciones de educación superior, como la enseñanza y el servicio público. Incluso, ya han aparecido algunos escalafones específicos con énfasis en la sustentabilidad o comparación docente, y sub-ránquines que permiten visualizar carreras específicas.

En general, los escalafones otorgan un peso preponderante a criterios como la producción científica de las universidades o el número de ganadores de premios Nobel que pertenecen a su planta académica; factores que, como el sesgo a favor de las publicaciones científicas en inglés, favorecen a las universidades anglosajonas en general (Ordorika y Rodríguez, 2010). Los ránquines también requieren de un alto grado de normalización en sus mediciones para poder comparar entre la gran diversidad de universidades en el mundo. Para abordar el desafío de considerar otros aspectos de impacto específicos como la sustentabilidad, han aparecido nuevos escalafones de especialidades y temas en el mundo. Por ejemplo, los ránquines regionales que permiten comparar a más universidades dentro de áreas con otros requisitos específicos para el área, en el caso de los ránquines latinoamericanos la producción científica mínima requerida es un $25 \%$ de la necesaria para formar parte del ranquin mundial.

Como resultado, en la mayoría de los escalafones mundiales solo unas 80 instituciones latinoamericanas figuran entre las mejores 1000 universidades, y muchas de ellas en solo alguno de los ránquines. Entre ellas, las instituciones de la región que en promedio se encuentran en las mejores posiciones están: la Universidad de Sao Paulo, la Universidad Federal de Campiñas en Brasil, las universidades Técnica Federico Santa María, U. de Chile y Pontificia U. Católica en Chile, las de los Andes y Javeriana en Colombia, la Universidad de Buenos Aires en Argentina y las universidades Nacional Autónoma (Unam) y Tecnológica de Monterrey de México; todas con una larga trayectoria y gran producción científica.

\section{La importancia de los escalafones}

Dentro de las definiciones de escalafón se encuentran varias que tratan de dar ideas sobre que son, considerando que estos hoy en día miden tanto información cualitativa (percepción) como cuantitativa (información bibliométrica). Son muchas las definiciones existentes, a continuación, se presentan dos definiciones que se acercan a las ideas centrales de los rankings.

"Los rankings son esencialmente compilaciones de información, proporcionadas de acuerdo a un conjunto de criterios para resaltar diferencias reales o percibidas en calidad" 
"Los rankings usan indicadores o métricas ponderadas para medir la actividad de la educación superior. El marcador agregado se expresa como un dígito simple, y las instituciones son posicionadas (ranqueadas) respecto a la universidad con mejor rendimiento".

"El benchmarking, que persigue la identificación de las mejores prácticas en otras organizaciones, con el objetivo de aprehenderlas y mejorar el rendimiento de un proceso o función determinada."

Vale la pena mencionar que los ránquines muestran la calidad de manera relativa y no absoluta, es decir, comparan los resultados con otras instituciones del mismo listado, de este modo facilita la entrega de información.

La importancia de los escalafones radica en cómo influye en las decisiones de distintos actores y agencias. Organismos de educación superior como directorios y consejos superiores analizan y se preocupan por el futuro de las instituciones y su prestigio, lo cual hace inevitablemente la revisión de estos. Los escalafones influyen en los siguientes motivos:

- Los estudiantes (y sus familias) que buscan acceder a las mejores opciones educativas (inversión económica).

- Los empleadores que utilizan las mediciones de calidad de los programas como filtro para sus prácticas de selección de personal.

- Los sistemas universitarios nacionales que son reconocidos con un mayor peso académico obtienen una reputación que se proyecta a diversos países.

- Los inversionistas ocupan los ránquines para invertir su dinero y, al mismo tiempo, asociar su imagen corporativa con el éxito de estas.

- Las universidades los utilizan para fines comerciales y de publicidad, así como para determinar estrategias institucionales en la captación de alumnos.

- Promueven una cultura de la calidad en el sistema de educación superior, al preocuparse de no perder el prestigio y caer lugares en los escalafones.

- Permiten tener una visión de la posición relativa relacionada con la calidad, el prestigio y la productividad de la universidad en la comunidad internacional, pudiendo ver sus resultados en un contexto global.

Frecuentemente se interpretan los escalafones como un reflejo de la calidad absoluta de las instituciones de educación superior o, por lo menos, como la mejor aproximación a ella; de modo que, su impacto suele ser importante tanto en términos económicos como políticos. Por ejemplo, la posición de las universidades públicas en los ránquines puede tener repercusiones en el monto del subsidio que reciben del gobierno, así como en el diseño de las políticas públicas de evaluación de la educación superior.

Estos también inciden en la elección de universidades por parte de los estudiantes, así como en el monto de las cuotas que están dispuestos a pagar. Inclusive, en el caso de Dinamarca, las autoridades migratorias utilizan uno de estos sistemas de clasificación de universidades para evaluar a los extranjeros que solicitan la residencia (The World Bank's Education for Global Development blog)

\section{Contextualizaciónenelmarcolatinoamericano}

Un elemento a considerar es el número de universidades en el mundo. En Latinoamérica el ranquin webometrics, que consideramos por ser el más amplio y extenso de todos, incorpora del orden de 26000 universidades e instituciones de educación superior; sin embargo, en este análisis se considera algunos institutos y organizaciones similares. La figura 1 nos muestra la información del ranquin por zonas geográficas.

Hace algunos años en los escalafones mundiales solo aparecían tres universidades, estos estaban limitados a las mejores 400 o 500 universidades. En la actualidad, con el aumento del número de universidades mencionadas, y con el esfuerzo y 


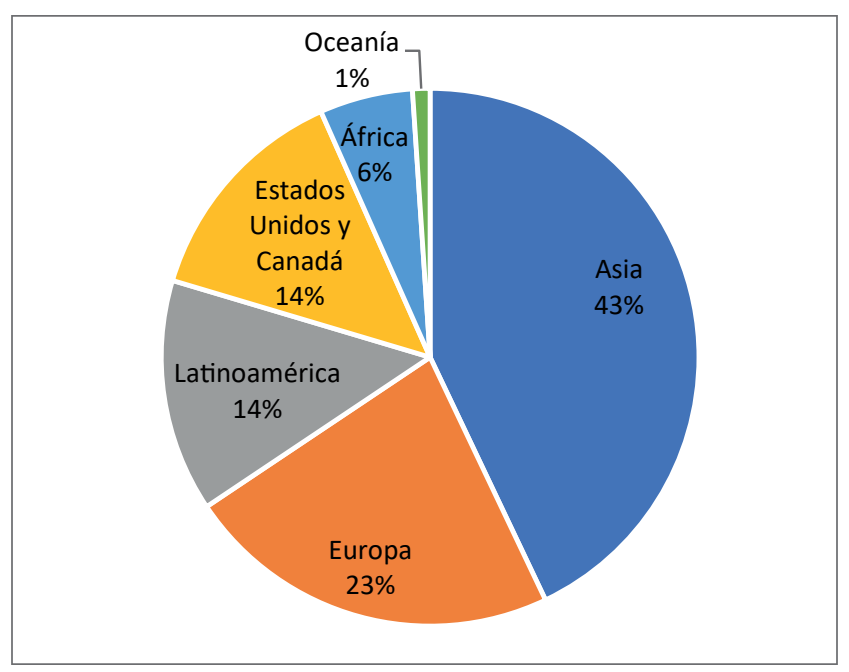

Figura 1. Número de universidades en el mundo por región. Fuente: elaboración propia con datos del ranquin Webometrics.

políticas para mejorar la participación, ahora aparecen alrededor de 90 universidades de la región en los escalafones mundiales, y más de 300 en los latinoamericanos.
El ranquin QS, el cual es el que incorpora más universidades latinoamericanas con 89 universidades latinas entre las 959 y con 394 en su ranquin latinoamericano, 40 de ellas chilenas, 89 brasileñas, 66 mexicanas y 52 colombianas; y el escalafón Times Higher Education incorpora a 47 universidades latinoamericanas entre las 1000 mejores del mundo.

Si consideramos la participación entre las mejores del mundo tenemos que, en general, la participación de la región es baja. Sin embargo, Brasil y Chile son los países de la región con más universidades entre las mejores del mundo. Sin considerar a QS, el cual es claramente un escalafón en el que la popularidad es una componente principal (40\% del total) entre Chile y Brasil se tiene entre el $72 \%$ al $85 \%$ de las mejores universidades de Latinoamérica.

Con respecto a la ponderación en tres de los escalafones más importantes sobre universidades en el mundo, Times Higher Education y QS son ingleses y durante varios años trabajaron juntos

Tabla 1. Número de universidades por región en el mundo

\begin{tabular}{cccc}
\hline Zona geográfica & Universidades & Población MM & U. por millón de habitantes \\
\hline Asia & 11398 & 4581 & 2.49 \\
Europa & 6021 & 735 & 8.19 \\
Latinoamérica & 3714 & 652 & 5.70 \\
Estados Unidos y Canadá & 3646 & 370 & 9.85 \\
África & 1488 & 1234 & 1.21 \\
Oceanía & 279 & 41 & 6.80 \\
Total & $\mathbf{2 6 5 4 6}$ & $\mathbf{7 6 1 3}$ & $\mathbf{3 . 4 9}$
\end{tabular}

Tabla 2. Número de universidades latinoamericanas mencionadas en distintos escalafones de universidades mundiales

\begin{tabular}{cccc}
\hline Ranking mundial & Univ. Mundo & Latinoamericanas & \% Mundo \\
\hline Times Higher Education & 1000 & 47 & $4.7 \%$ \\
QS & 959 & 89 & $9.3 \%$ \\
Shangai & 800 & 20 & $2.5 \%$ \\
US. News Best Global Universities & 1250 & 64 & $5.1 \%$ \\
CWUR Ranking & 1000 & 29 & $2.9 \%$ \\
Promedio & $\mathbf{1 0 0 2}$ & $\mathbf{5 0}$ & $5.0 \%$
\end{tabular}


Tabla 3. Ponderación de indicadores relacionados con Internacionalización en rankings de Universidades

\begin{tabular}{cccc}
\hline Indicador del ranquin & $\begin{array}{c}\text { Times Higher Education } \\
\text { World University } \\
\text { Rankings (THE) }\end{array}$ & $\begin{array}{c}\text { QS World University } \\
\text { Rankings }\end{array}$ & $\begin{array}{c}\text { US News Report on Best } \\
\text { Global Universities }\end{array}$ \\
\hline Reputación pares investigación & $18.0 \%$ & & $25.0 \%$ \\
Colaboración publicaciones/otras & $2.5 \%$ & $5.0 \%$ & $10.0 \%$ \\
Estudiantes internacionales & $2.5 \%$ & $5.0 \%$ & \\
Profesores internacionales & $23.0 \%$ & $10.0 \%$ & $35.0 \%$ \\
Ponderación total relacionada con & internacionalización & &
\end{tabular}

antes de que Times Higher Education decidiera mejorar los indicadores de evaluación de las universidades. El escalafón US News Report de Best Global Universities en Norteamérica es el que da una ponderación bastante alta, con un $35 \%$ relacionado con la colaboración e internacionalización de la universidad.

Si agregamos el indicador de reputación de pares académicos de QS, que puede relacionarse con la combinación entre desarrollo de la reputación internacional y algún grado de colaboración entre académicos o participación de académicos en eventos internacionales, el porcentaje del ranquin QS sube del $10 \%$ al $50 \%$ dado que este indicador pondera un $40 \%$ del escalafón.

El único ranquin internacional de gran reconocimiento es el ARWU (Academic Ranking of World Universities) de la Universidad de Shangai, conocido como el escalafón de Shangai. Este da mucha ponderación al prestigio al considerar

Tabla 4. Escalafón Times Higher de las 10 mejores universidades del mundo

\begin{tabular}{|c|c|c|c|c|c|c|c|c|c|}
\hline Rank & País & Universidad & Overall & Teaching & Research & Citations & $\begin{array}{l}\text { Industry } \\
\text { Income }\end{array}$ & $\begin{array}{l}\text { International } \\
\text { Outlook }\end{array}$ & $\begin{array}{l}\% \text { alumnos } \\
\text { extranjeros }\end{array}$ \\
\hline 1 & $\begin{array}{c}\text { United } \\
\text { Kingdom }\end{array}$ & University of Oxford & 96.0 & 91.8 & 99.5 & 99.1 & 67.0 & 96.3 & $40 \%$ \\
\hline 2 & $\begin{array}{l}\text { United } \\
\text { Kingdom }\end{array}$ & $\begin{array}{l}\text { University of Cambri- } \\
\text { dge }\end{array}$ & 94.8 & 92.1 & 98.8 & 97.1 & 52.9 & 94.3 & $37 \%$ \\
\hline 3 & $\begin{array}{l}\text { United } \\
\text { States }\end{array}$ & Stanford University & 94.7 & 93.6 & 96.8 & 99.9 & 64.6 & 79.3 & $23 \%$ \\
\hline 4 & $\begin{array}{l}\text { United } \\
\text { States }\end{array}$ & $\begin{array}{c}\text { Massachusetts Institute } \\
\text { of Technology }\end{array}$ & 94.2 & 91.9 & 92.7 & 99.9 & 87.6 & 89.0 & $34 \%$ \\
\hline 5 & $\begin{array}{l}\text { United } \\
\text { States }\end{array}$ & $\begin{array}{c}\text { California Institute of } \\
\text { Technology }\end{array}$ & 94.1 & 94.5 & 97.2 & 99.2 & 88.2 & 62.3 & $29 \%$ \\
\hline 6 & $\begin{array}{l}\text { United } \\
\text { States }\end{array}$ & Harvard University & 93.6 & 90.1 & 98.4 & 99.6 & 48.7 & 79.7 & $26 \%$ \\
\hline 7 & $\begin{array}{l}\text { United } \\
\text { States }\end{array}$ & Princeton University & 92.3 & 89.9 & 93.6 & 99.4 & 57.3 & 80.1 & $24 \%$ \\
\hline 8 & $\begin{array}{l}\text { United } \\
\text { States }\end{array}$ & Yale University & 91.3 & 91.6 & 93.5 & 97.8 & 51.5 & 68.3 & $20 \%$ \\
\hline 9 & $\begin{array}{l}\text { United } \\
\text { Kingdom }\end{array}$ & $\begin{array}{l}\text { Imperial College Lon- } \\
\text { don }\end{array}$ & 90.3 & 85.8 & 87.7 & 97.8 & 67.3 & 97.1 & $56 \%$ \\
\hline \multirow[t]{2}{*}{10} & $\begin{array}{l}\text { United } \\
\text { States }\end{array}$ & University of Chicago & 90.2 & 90.2 & 90.1 & 99.0 & 41.4 & 70.0 & $25 \%$ \\
\hline & $\begin{array}{l}\text { Promedio } \\
\text { Ind. }\end{array}$ & & 93.2 & 91.2 & 94.8 & 98.9 & 62.7 & 81.6 & $31 \%$ \\
\hline
\end{tabular}


premios Nobel, premios y medallas field, una alta ponderación a las revistas Science y Nature, y los artículos altamente citados de acuerdo con Clarivate Analytics.

En la tabla 4 se puede ver que las mejores universidades del mundo tienen un alto grado de internacionalización, con un $31 \%$ de sus alumnos extranjeros, con un máximo de $56 \%$ de alumnos extranjeros en el Imperial College London; ciertamente un mayor porcentaje de alumnos extranjeros colabora a que dos universidades inglesas estén en el tope de la tabla. Es posible

Tabla 5. Escalafón 2015 de Times Higher Education de las 10 mejores universidades Latinoamericanas. (En esta versión solo cuatro universidades Latinoamericanas aparecían)

\begin{tabular}{|c|c|c|c|c|c|c|c|c|}
\hline País & $\begin{array}{l}\text { Rank } 2015 \\
\text { Mundial }\end{array}$ & Name & Over all & Teaching & Research & Citations & $\begin{array}{l}\text { Industry } \\
\text { Income }\end{array}$ & $\begin{array}{l}\text { International } \\
\text { Outlook }\end{array}$ \\
\hline Brazil & $201-225$ & University of São Paulo & 44.3 & 51.6 & 51.6 & 32.3 & 40.1 & 25.3 \\
\hline Chile & $251-275$ & $\begin{array}{l}\text { Federico Santa María } \\
\text { Technical University }\end{array}$ & 40.2 & 13 & 10.1 & 99.7 & 28.1 & 48.7 \\
\hline Colombia & $251-275$ & $\begin{array}{l}\text { University of the Andes, } \\
\text { Colombia }\end{array}$ & 39.9 & 17.3 & 12.1 & 89.8 & 36.5 & 54.6 \\
\hline Brazil & $301-350$ & University of Campinas & 36.9 & 43.4 & 38.8 & 28 & 44.5 & 20.7 \\
\hline
\end{tabular}

Tabla 6. Ranking 2017 de Times Higher Education de las 10 mejores universidades latinoamericanas

\begin{tabular}{|c|c|c|c|c|c|c|c|c|}
\hline País & $\begin{array}{l}\text { Rank } \\
2017\end{array}$ & Name & Overall & Teaching & Research & Citations & $\begin{array}{l}\text { Industry } \\
\text { Income }\end{array}$ & $\begin{array}{l}\text { International } \\
\text { Outlook }\end{array}$ \\
\hline Brazil & $251-300$ & University of São Paulo & $43.5-46.2$ & 57.2 & 60.2 & 25.7 & 39.6 & 28.3 \\
\hline Brazil & $401-500$ & University of Campinas & $32.6-37.5$ & 44.9 & 39.6 & 28.0 & 46.5 & 24.1 \\
\hline Chile & $401-500$ & $\begin{array}{l}\text { Federico Santa María } \\
\text { Technical University }\end{array}$ & $32.6-37.5$ & 14.2 & 10.8 & 69.0 & 41.0 & 54.7 \\
\hline Chile & $401-500$ & $\begin{array}{c}\text { Pontifical Catholic University } \\
\text { of Chile }\end{array}$ & $32.6-37.5$ & 27.5 & 23.0 & 41.8 & 35.7 & 53.2 \\
\hline Colombia & $501-600$ & $\begin{array}{l}\text { University of the Andes, } \\
\text { Colombia }\end{array}$ & $27.6-32.5$ & 19.9 & 14.9 & 50.0 & 49.4 & 55.5 \\
\hline Chile & $501-600$ & University of Chile & $27.6-32.5$ & 25.4 & 18.4 & 36.9 & 32.1 & 46.7 \\
\hline Mexico & $501-600$ & $\begin{array}{l}\text { Monterrey Institute of } \\
\text { Technology }\end{array}$ & $27.6-32.5$ & 25.0 & 16.6 & 36.5 & 93.5 & 67.6 \\
\hline Mexico & $501-600$ & $\begin{array}{l}\text { National Autonomous } \\
\text { University of Mexico }\end{array}$ & $27.6-32.5$ & 36.8 & 33.0 & 17.8 & 60.2 & 37.2 \\
\hline Colombia & $501-600$ & $\begin{array}{c}\text { Pontifical Javeriana } \\
\text { University }\end{array}$ & $27.6-32.5$ & 15.8 & 8.0 & 63.7 & 32.8 & 44.0 \\
\hline Chile & $601-800$ & University of Concepción & $18.6-27.5$ & 15.5 & 13.0 & 20.8 & 56.4 & 46.2 \\
\hline Chile & $601-800$ & Diego Portales University & $18.6-27.5$ & 11.8 & 8.5 & 52.2 & 32.8 & 48.6 \\
\hline
\end{tabular}


notar que el promedio de alumnos extranjeros en las mejores universidades es muy alto, con un $31 \%$ versus el $4 \%$ promedio de 10 de las mejores universidades latinoamericanas.

Tabla 7. Escalafón 2019 de Times Higher Education de las 10 mejores universidades latinoamericanas

\begin{tabular}{|c|c|c|c|c|c|c|c|c|}
\hline País & $\begin{array}{l}\text { Rank } \\
2019\end{array}$ & Name & Overall & Teaching & Research & Citations & $\begin{array}{l}\text { Industry } \\
\text { Income }\end{array}$ & $\begin{array}{c}\text { International } \\
\text { Outlook }\end{array}$ \\
\hline Brazil & $251-300$ & $\begin{array}{l}\text { University of São } \\
\text { Paulo }\end{array}$ & $46.4-49.4$ & 55.9 & 53.5 & 37 & 39.5 & 32.7 \\
\hline Brazil & $401-500$ & $\begin{array}{l}\text { University of } \\
\text { Campinas }\end{array}$ & $37.1-41.6$ & 46.8 & 37.5 & 33.4 & 44.6 & 28.6 \\
\hline Chile & $401-500$ & $\begin{array}{l}\text { University of } \\
\text { Desarrollo }\end{array}$ & $37.1-41.6$ & 12.5 & 8.2 & 94.3 & 35.5 & 45.6 \\
\hline Chile & $401-500$ & $\begin{array}{l}\text { Diego Portales } \\
\text { University }\end{array}$ & $37.1-41.6$ & 13.2 & 10.3 & 90.1 & 34 & 51.2 \\
\hline Colombia & $501-600$ & $\begin{array}{l}\text { Pontifical Jave- } \\
\text { riana University }\end{array}$ & $33.5-37.0$ & 14.4 & 8.6 & 84 & 34.1 & 44.6 \\
\hline Chile & $601-800$ & $\begin{array}{l}\text { Federico Santa } \\
\text { María Technical } \\
\text { University }\end{array}$ & $26.0-33.4$ & 12.1 & 10.2 & 50.4 & 44.3 & 54.4 \\
\hline Colombia & $601-800$ & $\begin{array}{l}\text { University of the } \\
\text { Andes, Colom- } \\
\text { bia }\end{array}$ & $26.0-33.4$ & 23.3 & 13.4 & 49.2 & 36.4 & 51.7 \\
\hline Chile & $601-800$ & $\begin{array}{l}\text { University of } \\
\text { Chile }\end{array}$ & $26.0-33.4$ & 24.3 & 18 & 34.6 & 34 & 50.5 \\
\hline Brazil & $601-800$ & $\begin{array}{l}\text { Federal Univer- } \\
\text { sity of Minas } \\
\text { Gerais }\end{array}$ & $26.0-33.4$ & 29.3 & 16.8 & 42.5 & 38.3 & 25.4 \\
\hline Brazil & $601-800$ & $\begin{array}{l}\text { Federal Univer- } \\
\text { sity of Rio de } \\
\text { Janeiro }\end{array}$ & $26.0-33.4$ & 33.4 & 21.4 & 23.6 & 35.2 & 29 \\
\hline Mexico & 601-800 & $\begin{array}{l}\text { Monterrey Insti- } \\
\text { tute of Techno- } \\
\text { logy }\end{array}$ & $26.0-33.4$ & 22 & 16.1 & 39.8 & 73.5 & 59.9 \\
\hline Chile & 601-800 & $\begin{array}{l}\text { Pontifical Catho- } \\
\text { lic University of } \\
\text { Chile }\end{array}$ & $26.0-33.4$ & 25.5 & 24 & 44 & 39 & 56.9 \\
\hline Mexico & $601-800$ & $\begin{array}{l}\text { National Auto- } \\
\text { nomous Univer- } \\
\text { sity of Mexico }\end{array}$ & $26.0-33.4$ & 32.6 & 28.1 & 19.2 & 55.7 & 40.5 \\
\hline Brazil & $601-800$ & $\begin{array}{l}\text { Federal Universi- } \\
\text { ty of Rio Grande } \\
\text { do Sul }\end{array}$ & $26.0-33.4$ & 31.7 & 14.1 & 40.5 & 48.1 & 24.9 \\
\hline Brazil & $601-800$ & $\begin{array}{l}\text { Federal Univer- } \\
\text { sity of São Paulo } \\
\text { (Unifesp) }\end{array}$ & $26.0-33.4$ & 33.9 & 19.7 & 45.8 & 35 & 27 \\
\hline
\end{tabular}


Tabla 8. Evolución del indicador de internacionalización (International Outlook) del escalafón Times Higher Education, evolución 2015 a 2019

\begin{tabular}{cccccc}
\hline País & $\begin{array}{c}\text { Universidad (evolución de indicador de } \\
\text { internacionalización) }\end{array}$ & $\mathbf{2 0 1 5}$ & $\mathbf{2 0 1 7}$ & $\mathbf{2 0 1 9}$ & $\begin{array}{c}\text { \% de alumnos } \\
\text { extranjeros 2019 }\end{array}$ \\
\hline Brazil & $\quad$ University of São Paulo & 25.3 & 28.3 & 32.7 & $4 \%$ \\
Chile & Federico Santa María Technical Univer- & 48.7 & 54.7 & 54.4 & $3 \%$ \\
Solombia & University of the Andes, Colombia & $\mathbf{5 4 . 6}$ & 55.5 & 51.7 & $2 \%$ \\
Brazil & $\quad$ University of Campinas & 20.7 & 24.1 & 28.6 & \\
Chile & Pontifical Catholic University of Chile & & 53.2 & 56.9 & $7 \%$ \\
Chile & $\quad$ University of Chile & 46.7 & 50.5 & $6 \%$ \\
Mexico & Monterrey Institute of Technology & & $\mathbf{6 7 . 6}$ & $\mathbf{5 9 . 9}$ & $11 \%$ \\
Mexico & National Autonomous University of & & 37.2 & 40.5 & $6 \%$ \\
Colombia & $\quad$ Pontifical Javeriana University & & 44.0 & 44.6 & $1 \%$ \\
Chile & Diego Portales University & & 48.6 & 51.2 & $2 \%$ \\
Brazil & Federal University of Minas Gerais & & 22.7 & 25.4 & $2 \%$ \\
Brazil & Federal University of Rio de Janeiro & & 27.9 & 29.0 & $2 \%$ \\
& $\quad$ Promedio & 42.5 & 43.8 & $4 \%$
\end{tabular}

Tabla 9. Universidades colombianas por orden de indicador de internacionalización (International Outlook) del escalafón Times Higher Education, 2019

\begin{tabular}{|c|c|c|c|c|c|c|c|}
\hline Rank THE & $\begin{array}{l}\text { Universidad (por } \\
\text { orden de indicador de } \\
\text { internacionalización) }\end{array}$ & Overall & Teaching & Research & Citations & $\begin{array}{l}\text { Industry } \\
\text { Income }\end{array}$ & $\begin{array}{l}\text { International } \\
\text { Outlook }\end{array}$ \\
\hline $601-800$ & $\begin{array}{l}\text { University of } \\
\text { the Andes }\end{array}$ & $26.0-33.4$ & 23.3 & 13.4 & 49.2 & 36.4 & 51.7 \\
\hline $1001+$ & Del Rosario University & $9.8-18.9$ & 15.6 & 8.9 & 18.1 & 34.2 & 45.6 \\
\hline $501-600$ & $\begin{array}{c}\text { Pontifical Javeriana } \\
\text { University }\end{array}$ & $33.5-37.0$ & 14.4 & 8.6 & 84.0 & 34.1 & 44.6 \\
\hline $801-1000$ & $\begin{array}{l}\text { Industrial University } \\
\text { of Santander (UIS) }\end{array}$ & $19.0-25.9$ & 13.0 & 8.2 & 32.3 & 34.5 & 39.6 \\
\hline $1001+$ & $\begin{array}{l}\text { University of Antio- } \\
\text { quia }\end{array}$ & $9.8-18.9$ & 16.4 & 20.4 & 13.0 & 40.2 & 38.0 \\
\hline $1001+$ & $\begin{array}{l}\text { National University } \\
\text { of Colombia }\end{array}$ & $9.8-18.9$ & 19.3 & 14.7 & 13.0 & 34.2 & 36.2 \\
\hline $1001+$ & $\begin{array}{l}\text { Pontifical Bolivarian } \\
\text { University (UPB)- } \\
\text { Medellín }\end{array}$ & 9.8-18.9 & 16.0 & 8.0 & 4.8 & 36.3 & 35.1 \\
\hline
\end{tabular}


En el caso de Colombia solo la Universidad de los Andes tiene un índice de internacionalización superior a 50 puntos relativos, entre las 1250 mejores universidades del mundo de acuerdo con el ranquin Times Higher Education.

\section{Conclusión}

A pesar de la creciente importancia de la internacionalización de las universidades, en Latinoamérica, según los ránquines de universidades internacionales, estamos al debe.

La internacionalización de las universidades es considerada de manera meridiana en los escalafones internacionales de universidades. Si bien es cierto que en todos ellos se consideran diferentes indicadores, uno de los más comunes es el número de alumnos extranjeros y de profesores extranjeros que se tiene en una universidad. Sin embargo, las universidades latinoamericanas en general tienen indicadores de internacionalización muy bajos, salvo el Tecnológico de Monterrey y la Universidad de Buenos Aires. En términos relativos a la internacionalización de universidades en el mundo, el Tecnológico de Monterrey bajó desde 67.6 puntos a 59.9 en el periodo 2017 a 2019, por lo que otras universidades fuera de la región han ido mejorando sus niveles de internacionalización en una escala mayor que las universidades en Latinoamérica, especialmente en Asia y Europa.

Entre las mejores universidades de la región, de acuerdo con los ránquines, el indicador de internacionalización ha mejorado durante los últimos años, pero estos permiten corroborar que universidades en otras regiones han mejorado aún más; por lo que es notable que los esfuerzos en la región no son suficientes en esta materia.

Si quisiéramos definir cuál sería un indicar adecuado podríamos indicar el \% mínimo de alumnos extranjeros de la evaluación de QS stars (que no es un escalafón sino una medida de adopción de mejores prácticas en la educación superior) es de un $5 \%$ de alumnos extranjeros para tener cinco estrellas. Por tanto, para universidades que buscan la excelencia este debería ser el indicador meta.

Existen grandes oportunidades para la movilidad intrarregional en Latinoamérica, por el momento el mayor interés de los alumnos de la región está en viajar a Estados Unidos y Europa, además de que se detecta que hay un creciente interés por Asia.

La movilidad de alumnos considera el estudio de toda la carrera como es común en la Universidad de Buenos Aires, pero también existen cada vez más programas de un año o un semestre, así como estadías cortas de una a varias semanas; pero son las estadías más cortas de dos o tres semanas las que representan una creciente oportunidad de internacionalización en las universidades de la región.

La reputación internacional es otro indicador importante para apoyar la internacionalización. En la actualidad la mayoría de los profesores de intercambio, alumnos de intercambio y las oficinas de asuntos internacionales de las universidades, así como investigadores que colaboran internacionalmente, suelen revisar los antecedentes en los escalafones.

Los beneficios de los acuerdos internacionales son claros, pero son difíciles de poner en marcha, desarrollar y mantener en el tiempo estos programas. La laboriosidad de formar asociaciones universitarias significa que solo aquellos identificados como capaces de soportar a largo plazo realmente deberían ser perseguidos. Desarrollar relaciones exitosas lleva mucho tiempo, desde comprender la cultura y los objetivos de las instituciones de cada uno hasta garantizar la compatibilidad en términos de objetivos, programas, estándares, entre otros aspectos.

En este sentido, es importante asegurarse de que cualquier asociación universitaria pueda adaptarse y sobrevivir en circunstancias cambiantes. La investigación ha demostrado que la mejor manera de formar estas asociaciones a menudo proviene de un enfoque personal, de personas que potencian y desarrollan la colaboración. 
Los académicos e investigadores, una vez más, son los mejores activos y promotores de la colaboración internacional, siendo importante reconocerlos y apoyarlos, al igual que las oficinas de asuntos internacionales.

Para las universidades que aspiran a ser consideradas entre las mejores del mundo o que buscan mejorar su impacto en la sociedad les es necesario desarrollar una estrategia de internacionalización. En este sentido, los escalafones internacionales proporcionan indicadores básicos de internacionalización y la oportunidad de seguirlos y compararlos con otras universidades del mundo.

\section{Referencias}

Abbas, S. y Yousafzai, M. (2018). Internationalization of Universities: Challenges, Threats and Opportunities for Third World Countries. The Dialogue, 10(4), 378-389.

Altbach, P. (2011). The past, present and future of the research university. En P. Altbach y J. Salmi (eds.), The Road to Academic Excellence: The Making of World-Class Research Universities (pp. 11-32). Washington: The World Bank. https://doi. org/10.1596/9780821388051_CH01

Huang, F. (2018). The benefits and risks of HE internationalization. University World News. Recuperado de https://www.universityworldnews.com/ post.php?story $=20180508094144222$

Hudzik J. (2011). Internacionalización Integral, del concepto a la acción. Recuperado de http://obiret-iesalc.udg.mx/sites/default/files/publicaciones/ internacionalizacion_integral._del_concepto_a_ la_accion._resumen_ejecutivo._hudzik.pdf
Knight, J. (1993). Internationalization: management strategies and issues. International Education Magazine, 9(1), 21-22. https://doi.org/10.6017/ ihe.2018.95.10715

Knight, J. y Wit, H. (2008). Internationalization of Higher Education, Past and Future. International Higher Education, 95, 2-4.

Marginson, S. (2013). Nation-States, Educational Traditions and the WCU Project. En J. C. Shin, y B. M. Kehm (eds.) Institutionalization of World-Class University in Global Competition (pp. 59-77). Londres: Springer. https://doi. org/10.1007/978-94-007-4975-7_5

Ordorika, I. y Rodríguez, R. (2010). El ranking Times en el mercado del prestigio universitario. Perfiles Educativos, 32(129), 8-29. https://doi.org/10.22201/ iisue.24486167e.2010.129.18918

Reynolds, J. (2018). The Benefits of the Internationalisation of Higher Education. Recuperado de https://www.maastrichtuniversity.nl/blog/2018/06/ item-benefits-internationalisation-higher-education

Salmi, J. (2009). The Challenge of Establishing World-Class Universities. Recuperado de https://siteresources. worldbank.org/EDUCATION/Resources/278200 -1099079877269/547664-1099079956815/547 670-1237305262556/WCU.pdf

Simmons, R. (18 de enero de 2003). Striving for exceIlence: How to make a world-class university. South China Morning Post, 15.

Studiasi (2017). La internacionalización de las Universidades, una estrategia necesaria. Recuperado de https://www.universidadsi.es/la-internacionalizacion-las-universidades-una-estrategia-necesaria/

Zha, Q. (2003). Internationalization of Higher Education. Policy Futures in Education, 1(2), 248-270. https://doi.org/10.2304/pfie.2003.1.2.5

\section{(C) $(1) \Theta$}

